\section{Modalidades de gestão de serviços no Sistema Único de Saúde: revisão narrativa da produção científica da Saúde Coletiva no Brasil (2005-2016)}

\author{
Health services management modalities in the \\ Brazilian Unified National Health System: \\ a narrative review of research production \\ in Public Health (2005-2016)
}

Modalidades de gestión de servicios de salud en el Sistema Único Salud de Brasil: revisión narrativa de la producción científica de la Salud Colectiva (2005-2016)

\begin{abstract}
Resumo
O presente trabalho busca sistematizar tendências e prioridades das abordagens teórico-conceituais e das investigações empíricas sobre modalidades específicas de gestão de serviços no âmbito do Sistema Único de Saúde no Brasil. Foi realizada uma revisão narrativa da literatura que identificou, em 33 publicações, a localização e a natureza dos serviços, os modelos de gestão, os procedimentos metodológicos e os desfechos dos estudos. A produção trata principalmente de aspectos conceituais, legais e práticas gerenciais dos modelos, além de abordar contratos, compras, recursos humanos, financiamento e mecanismos de controle. Concluiu-se que a literatura analisada é restrita, concentrada no Estado de São Paulo, com baixa diversidade de teorias e fragilidades de aportes metodológicos, sendo inconclusiva quanto à superioridade de um modelo de gestão sobre outro. São fundamentais novas pesquisas avaliativas capazes de comparar os diferentes modelos e julgar seus desempenhos e efeitos na qualidade da assistência prestada, na saúde da população e na organização do sistema de saúde.
\end{abstract}

Gestão em Saúde; Administração de Serviços de Saúde;

Sistema Único de Saúde

\begin{abstract}
Antonio Franco Ravioli 1
Patrícia Coelho De Soárez 1

Mário César Scheffer 1
\end{abstract}

doi: 10.1590/0102-311X00114217

\author{
Correspondência \\ A. F. Ravioli \\ Departamento de Medicina Preventiva, Faculdade de Medicina, \\ Universidade de São Paulo. \\ Av. Dr. Arnaldo 455, 2o andar, São Paulo, SP 01246-903, Brasil. \\ aravioli@gmail.com \\ 1 Faculdade de Medicina, Universidade de São Paulo, São Paulo, \\ Brasil.
}




\section{Introdução}

Os entes federativos que compõem o Sistema Único de Saúde (SUS), para cumprir a obrigação constitucional de assegurar assistência em saúde à população, podem gerir, manter e prestar serviços de forma direta ou indireta, inclusive mediante a participação complementar da iniciativa privada ${ }^{1}$.

Os estabelecimentos de saúde que atendem usuários do SUS exibem múltiplas composições organizacionais, formatos de administração e modelos de gestão, modificados ao longo do tempo por meio de reformas administrativas, interpretações jurídicas, decisões políticas e legislações complementares federal, estaduais e municipais.

Além de serviços de saúde próprios da administração direta da União, estados e municípios, o poder público delega ou transfere a titularidade ou a execução de serviços do SUS para a administração indireta, que pode ter diferentes personalidades jurídicas, entre elas as autarquias, fundações, empresas públicas e sociedades de economia mista. Para o mesmo fim de prestar assistência em saúde, o gestor público pode optar pela administração privada complementar, ao firmar contratos ou convênios com organizações sociais (OS), associações civis sem fins lucrativos, fundações privadas ou empresas privadas 2 .

A produção científica sobre gestão dos serviços de saúde no Brasil 3 tem tratado da orientação da gestão de hospitais e unidades públicas, das práticas de gestores, da natureza e controle dos convênios, dos processos de trabalho e da percepção de usuários, principalmente por meio de relatos, ensaios, estudos de caso e pesquisas avaliativas. Quanto à gestão do sistema de saúde, os estudos até agora abordaram, dentre outros temas, a gestão participativa, o financiamento, a regulação e a avaliação de serviços 4 .

Há, no entanto, uma importante lacuna sobre as características, o funcionamento e a eficácia dos arranjos e modelos em curso no interior do sistema público de saúde.

A presente revisão foca a produção de Saúde Coletiva, na subárea Política, Planejamento e Gestão em Saúde, visando sistematizar tendências e prioridades das abordagens teórico-conceituais e das investigações empíricas sobre modalidades específicas de gestão de serviços no âmbito do SUS.

Ao agregar evidências de pesquisas sobre dimensões que caracterizam, aproximam ou diferenciam os modelos destacados na literatura, espera-se contribuir para a compreensão dos desafios atuais e para o aprimoramento da gestão dos serviços de saúde.

\section{Metodologia}

Foi realizada uma revisão narrativa da literatura, que busca fornecer sínteses da produção científica anteriormente publicada sobre determinado tema, sempre que a questão de pesquisa não seja tão específica a ponto de ser abordada com o desenho de revisão sistemática 5 .

Foi levantada a produção nacional no campo da Saúde Coletiva sobre modalidades de gestão de serviços de saúde no âmbito do SUS. Com o propósito de aumentar a reprodutibilidade e a transparência, a revisão empregou métodos explícitos e informou as bases de dados eletrônicos, termos utilizados na estratégia de busca, estratégia de rastreamento, critérios de elegibilidade, de extração de dados e abordagem de síntese.

O levantamento bibliográfico, realizado por um revisor (A.F.R.), ocorreu no período de 1o a 29 de maio de 2017. Incluiu as bases MEDLINE (via PubMed), EMBASE, Literatura Latino-americana e do Caribe em Ciências da Saúde (LILACS), Scientific Electronic Library Online (SciELO) e Google Scholar. Foram considerados os trabalhos publicados de janeiro de 2005 a dezembro de 2016. Estudos relevantes adicionais foram identificados em outras fontes, com base nas listas de referências de estudos obtidas na busca eletrônica.

O recorte temporal foi definido considerando-se o período posterior ao marco legal de uma das modalidades possíveis de gestão, as Parcerias Público-Privadas (PPP), regulamentadas pela Lei no 11.079/2004 6. Com isso, buscou-se, no período analisado, contemplar produções sobre as principais alternativas de gestão existentes.

Nas estratégias de buscas foram utilizados termos de indexação específicos (Medical Subject Headings - MeSH; Descritores em Ciências da Saúde - DeCS). Para o MEDLINE e EMBASE foram 
incluídos os seguintes termos: "State Reform", "Reform of State", "Models of Health Sector Reform", "Health Sector Reform", "Organizational Models", "Health Organizations" e "Public-Private Partnerships”. A busca também foi realizada com o filtro de data de publicação de 2005 a 2016 e com restrição para "Brazil".

Nas bases LILACS e SciELO foram utilizados os termos para busca: "Reforma do Estado", "Reforma do Setor Saúde", "Modelos de Reforma em Saúde", "Modelos Organizacionais", "Organizações de Saúde", "Parceria Público-Privada", "Modelos de Gestão", "Gestão de Serviços de Saúde", "Gestão do Sistema Único de Saúde”. Em ambas, após a busca, selecionou-se o Brasil como o país relativo ao assunto.

Para Google Scholar, utilizou-se o sistema de busca específico desta base de dados. Além disso, foi realizada busca manual das referências citadas nos artigos selecionados nas bases anteriores. Foram obtidas publicações valendo-se dos seguintes termos: "Administração Direta", "Organização Social", "Fundação Estatal", "Fundação Pública”, "Parceria Público Privada”, "PPPs", "Empresa Brasileira de Serviços Hospitalares". Os termos livres foram incluídos para aumentar a sensibilidade da estratégia de busca e para alcançar capítulos de livros, além de artigos científicos.

Dois revisores independentes (A.F.R., P.C.D.S.) leram os títulos e resumos das publicações identificadas e, na segunda etapa, leram os textos na íntegra. Foram consideradas elegíveis para análise as produções científicas (artigos científicos, livros, capítulos de livros, teses/dissertações e relatórios) que abordassem a apresentação, descrição ou discussão de características de pelo menos um modelo ou formato de gestão de serviços do SUS.

Inicialmente, foram eliminadas todas as publicações que não tratassem de assuntos ligados à saúde. Em seguida, foram excluídas as publicações que tratavam do sistema de saúde, mas de assuntos não relacionados a modalidades de gestão de serviços, como descentralização, planejamento, protocolos clínicos, satisfação e participação de usuários, recursos humanos e processos de trabalho. Os artigos sobre serviços de assistência suplementar ou gestão de planos e seguros de saúde não foram incluídos.

Dois revisores independentes (A.F.R., P.C.D.S.) extraíram os dados (localização do objeto, modelo de gestão, desfechos, desenho da pesquisa, fonte dos dados, tipo de análise, número de participantes) das publicações selecionadas. No caso de discordância, a arbitragem foi realizada por um terceiro revisor (M.C.S.) e, então, as publicações agrupadas e categorizadas.

\section{Resultados}

Identificou-se 341 registros sobre o tema e, após a exclusão das duplicatas, foram rastreados 308 registros. Ao final da leitura na íntegra de 59 publicações, 33 delas foram incluídas na análise (Figura 1).

Foram considerados 27 artigos científicos, três relatórios, dois capítulos de livros e uma tese. Dentre os artigos, $23(85 \%$; $23 / 27)$ foram publicados em periódicos indexados e quatro $(15 \% ; 4 / 27)$ em fontes não indexadas. Em relação aos indexados, são os seguintes os periódicos que mais publicaram artigos que integram a revisão: Ciência er Saúde Coletiva (cinco artigos), Revista de Saúde Pública (quatro), Cadernos de Saúde Pública (três) e Einstein (dois artigos).

A Tabela 1 resume a localização, tipos de serviços, modelos de gestão analisados e desfechos dos estudos incluídos. Os trabalhos que mencionaram modelos específicos de gestão de serviços de saúde no SUS abordaram principalmente Organização Social de Saúde - OSS (61\%; 20/33), Administração Direta (30\%; 10/33), PPP (30\%; 10/33) e Fundação Estatal (12\%; 4/33). Em 11 estudos (33\%; 11/33) foram abordados mais de um modelo de gestão ou comparação entre eles.

Dos 33 trabalhos incluídos, 18 publicações (54\%; 16/33) analisaram a gestão de serviços localizados no Estado de São Paulo ligados à Secretaria Estadual de Saúde (SES) ou a alguma Secretaria de Saúde municipal. Há dois estudos $(6 \% ; 2 / 33)$ sobre equipamentos geridos no Mato Grosso e um estudo (3\%; 1/33) analisou o modelo PPP nos seguintes locais: Bahia, Ceará, Distrito Federal, Espírito Santo, Mato Grosso, Rio de Janeiro, Roraima, São Paulo, Belo Horizonte (Minas Gerais), Manaus (Amazonas), cidade de São Paulo e Sorocaba (São Paulo).

Quanto aos serviços de saúde que compõem os estudos da literatura analisada, constam unidades e hospitais públicos geridos por Secretarias Estaduais e Municipais de Saúde, além de hospitais privados e universitários. Em 13 artigos (39\%; 13/33) foram estudados exclusivamente hospitais; dois 
Figura 1

Fluxograma da seleção de estudos da revisão.

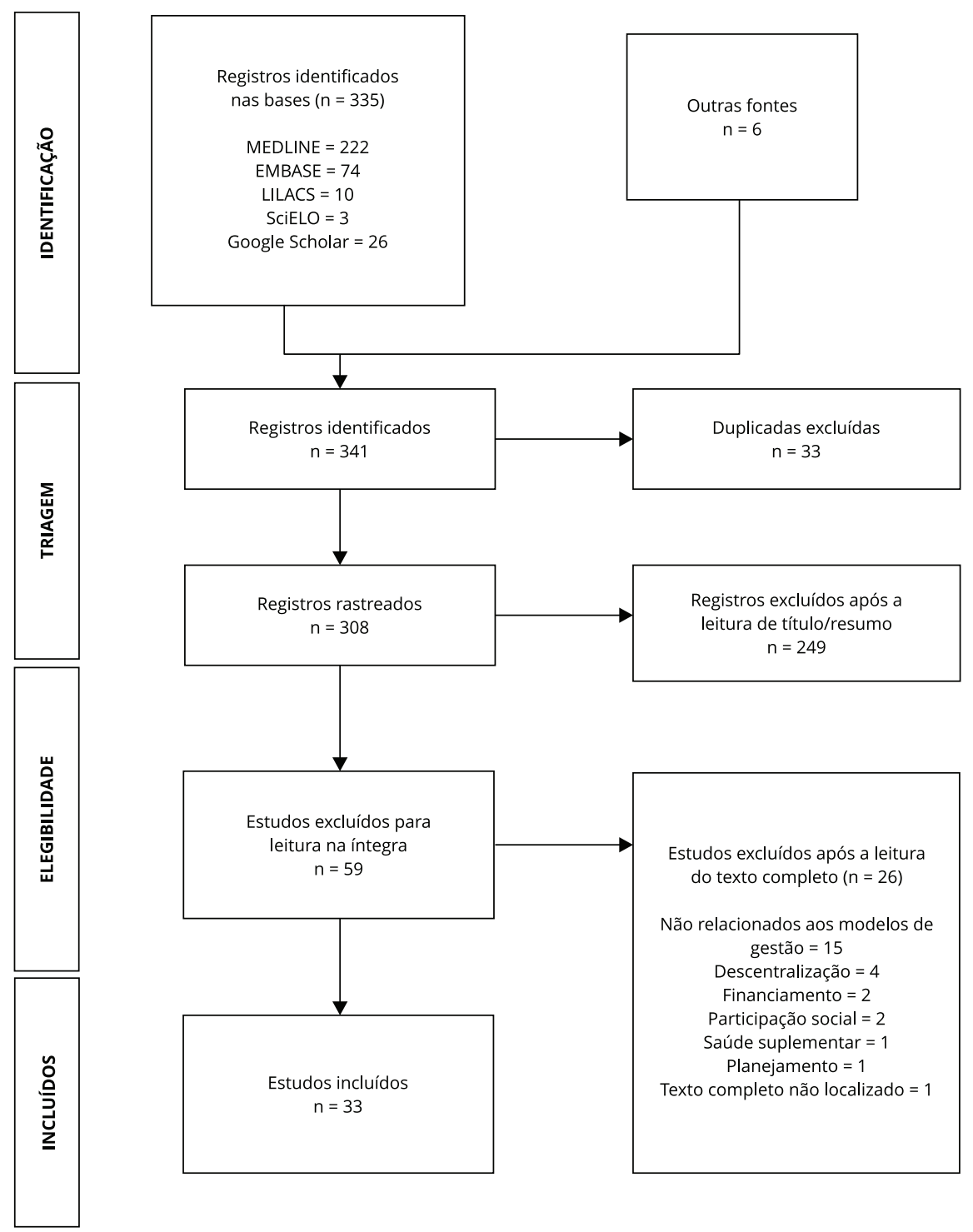

(6\%; 2/33) analisaram hospitais e outras unidades de saúde; um artigo (3\%; 1/33) analisou unidade de saúde não hospitalar. E, ainda, um dos estudos (3\%; 1/33), de abrangência nacional, abordou estabelecimentos de saúde públicos e privados em geral. As demais publicações $(48 \%$; 16/33) não trataram especificamente de serviços de saúde.

Com relação aos desfechos observados nos trabalhos, quatro temas, dentre outros, são mais tratados na literatura revisada: recursos humanos, contratos e compras, mecanismos de controle, e financiamento.

Quanto às abordagens metodológicas, os desenhos de pesquisa mais frequentes foram estudos descritivos (36\%; 12/33), pesquisa qualitativa $(36 \% ; 12 / 33)$ e estudos de caso $(33 \% ; 11 / 33)$. Em relação às análises, prevaleceram aquelas do tipo comparativa (33\%; 11/33), descritiva $(33 \% ; 11 / 33)$ e de 
Tabela 1

Publicações selecionadas segundo modelos de gestão, localização, tipos de serviços/instituições de saúde e desfechos.

\begin{tabular}{|c|c|c|c|c|}
\hline Modelos de gestão & Estudo & Localização & Serviços de saúde & Desfechos \\
\hline \multirow[t]{10}{*}{$\begin{array}{l}\text { Organização Social de Saúde } \\
\text { (OSS) }\end{array}$} & $\begin{array}{l}\text { Costa e Silva et al. } \\
\qquad(2016) 27\end{array}$ & $\begin{array}{l}\text { Cidade do Rio de } \\
\text { Janeiro }\end{array}$ & $\begin{array}{l}\text { SMS, atenção primária } \\
\text { à saúde }\end{array}$ & $\begin{array}{l}\text { Contratualização, regulação, } \\
\text { transparência pública e controle social }\end{array}$ \\
\hline & Pacheco et al. (2016) 40 & Pernambuco & SES, SMS & $\begin{array}{c}\text { Gestão estadual do SUS e o papel } \\
\text { das OSS; influências, decisões } \\
\text { e questionamentos quanto à } \\
\text { implementação, eficiência e autonomia } \\
\text { gerencial das OSS }\end{array}$ \\
\hline & Romano et al. (2015) 41 & Mato Grosso & SES & $\begin{array}{c}\text { Credenciamento, habilitação, } \\
\text { contratualização, pagamento, controle } \\
\text { e avaliação da SES }\end{array}$ \\
\hline & $\begin{array}{l}\text { Contreiras \& Matta } \\
\qquad(2015)^{7}\end{array}$ & São Paulo & SMS & $\begin{array}{c}\text { Privatização da gestão: marcos } \\
\text { legais das OSS, contratos de gestão, } \\
\text { convênios, caracterização das } \\
\text { entidades contratadas, regulação e } \\
\text { funcionamento }\end{array}$ \\
\hline & $\begin{array}{c}\text { Rodrigues \& } \\
\text { Spagnuolo (2014) } 42\end{array}$ & Botucatu (São Paulo) & $\begin{array}{l}\text { Fundação UNI, atenção } \\
\text { primária à saúde }\end{array}$ & $\begin{array}{c}\text { Processo de implantação, contrato de } \\
\text { gestão, limitações dos mecanismos de } \\
\text { controle }\end{array}$ \\
\hline & $\begin{array}{l}\text { Camargo et al. } \\
\qquad \text { (2013) } 34\end{array}$ & São Paulo & SES & $\begin{array}{c}\text { Controle, grau de transparência, } \\
\text { interatividade e acessibilidade aos } \\
\text { portais de informação }\end{array}$ \\
\hline & $\begin{array}{l}\text { La Forgia \& Harding } \\
\text { (2009) } 36\end{array}$ & São Paulo & 24 hospitais & $\begin{array}{c}\text { Eficiência de gestão, qualidade, custo e } \\
\text { gestão de recursos humanos }\end{array}$ \\
\hline & $\begin{array}{l}\text { Sano \& Abrucio } \\
\text { (2008) } 19\end{array}$ & São Paulo & SES & $\begin{array}{l}\text { Desempenho, responsabilização dos } \\
\text { gestores, controle de resultados }\end{array}$ \\
\hline & $\begin{array}{l}\text { Barata \& Mendes } \\
\qquad(2007) 43\end{array}$ & São Paulo & SES & Eficiência e qualidade \\
\hline & $\begin{array}{l}\text { Carneiro Junior \& Elias } \\
\qquad(2006) 35\end{array}$ & $\begin{array}{l}\text { Região Metropolitana } \\
\text { de São Paulo }\end{array}$ & 2 hospital municipais & $\begin{array}{c}\text { Modelo gerencial, equidade na } \\
\text { prestação de serviços e controle } \\
\text { público }\end{array}$ \\
\hline \multirow[t]{5}{*}{ Parceria Público-Privada (PPP) } & Cabral et al. (2016) 33 & Bahia & 1 hospital & $\begin{array}{c}\text { Relação público-privado e mecanismos } \\
\text { de viabilização de projetos }\end{array}$ \\
\hline & $\begin{array}{l}\text { Barbosa \& Malik } \\
\qquad(2015) 30\end{array}$ & $\begin{array}{c}\text { Bahia, Ceará, Espírito } \\
\text { Santo, Distrito Federal, } \\
\text { Mato Grosso, Rio de } \\
\text { Janeiro, Rondônia e } \\
\text { São Paulo. Cidades de } \\
\text { Belo Horizonte (Minas } \\
\text { Gerais), São Paulo, } \\
\text { Sorocaba (São Paulo) } \\
\text { e Manaus (Amazonas) }\end{array}$ & $\begin{array}{l}36 \text { hospitais, } 328 \text { UBS, } 4 \\
\text { centrais de diagnósticos } \\
\text { por imagem, } 4 \text { centros } \\
\text { de apoio logístico e } 1 \\
\text { indústria farmacêutica }\end{array}$ & $\begin{array}{l}\text { Investimentos previstos e estágios de } \\
\text { implantação dos projetos }\end{array}$ \\
\hline & $\begin{array}{l}\text { Melo \& Almeida } \\
\qquad(2014) 44\end{array}$ & Rio Grande do Norte & $\begin{array}{c}\text { Hospital universitário } \\
\text { administrado pela } \\
\text { EBSERH }\end{array}$ & $\begin{array}{l}\text { Estrutura organizacional (burocracia e } \\
\text { inovação do ambiente), conteúdo das } \\
\text { metas a serem alcançadas e controle } \\
\text { público sobre o setor privado }\end{array}$ \\
\hline & Mendes et al. (2014) 45 & Cidade de São Paulo & Hospital privado (HIAE) & $\begin{array}{c}\text { Remuneração e gastos hospitalares por } \\
\text { tipo de procedimento }\end{array}$ \\
\hline & $\begin{array}{l}\text { Anker \& Pereira } \\
\text { (2013) } 20\end{array}$ & $\mathrm{NI}$ & $\begin{array}{c}10 \text { hospitais e } 2 \text { unidades } \\
\text { de saúde }\end{array}$ & $\mathrm{NI}$ \\
\hline
\end{tabular}

(continua) 
Tabela 1 (continuação)

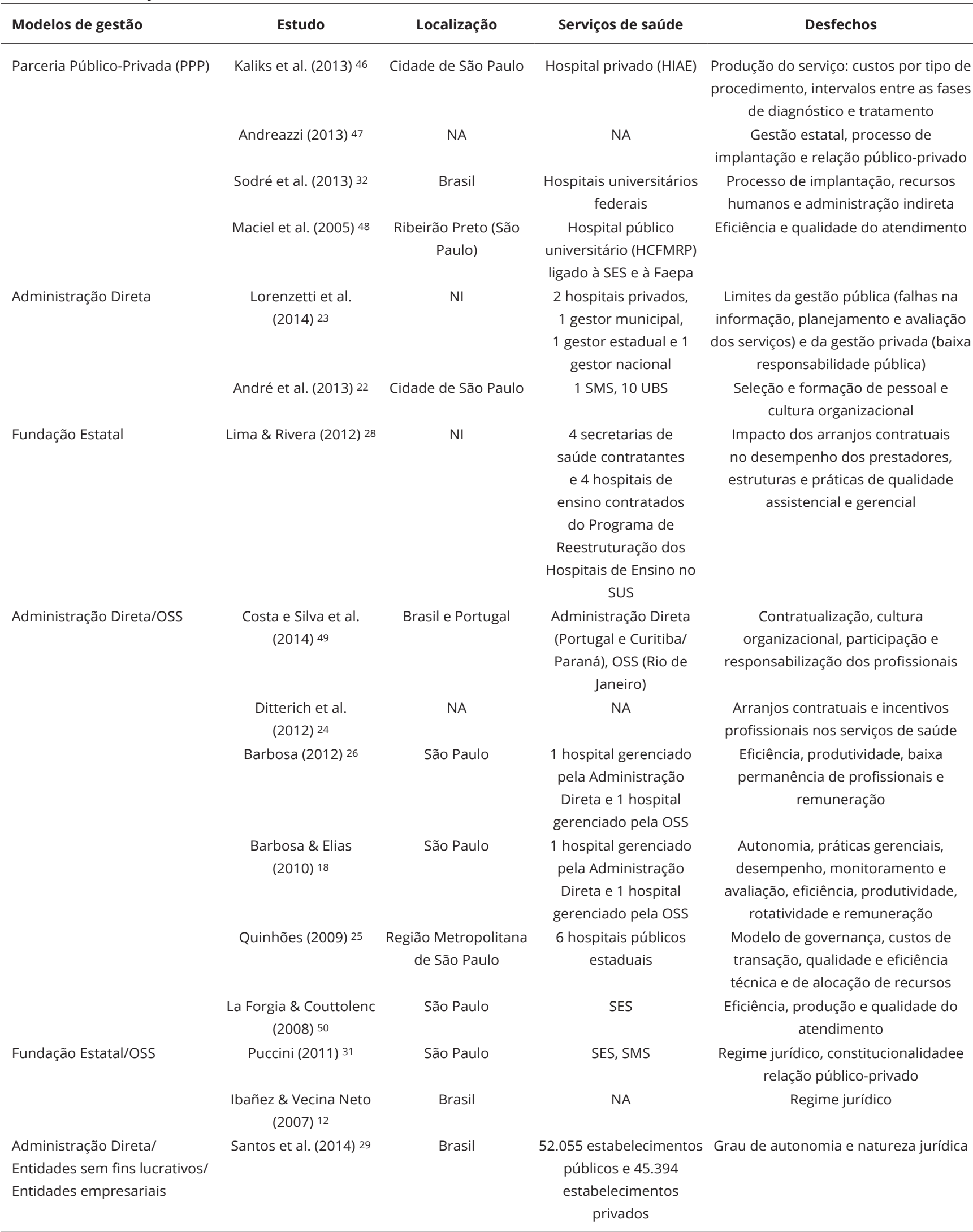

(continua) 
Tabela 1 (continuação)

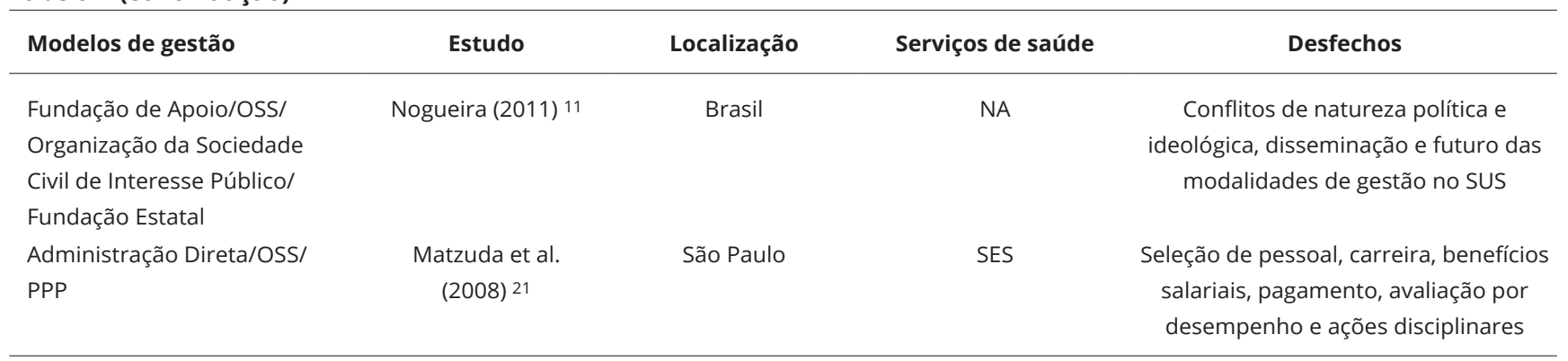

EBSERH: Empresa Brasileira de Serviços Hospitalares; Faepa: Fundação de Apoio ao Ensino, Pesquisa e Assistência do HCFMRP da Universidade de São Paulo; HCFMRP: Hospital das Clínicas da Faculdade de Medicina de Ribeirão Preto; HIAE: Hospital Israelita Albert Eisntein; NA: não aplicável; NI: não informado; SES: Secretaria Estadual de Saúde; SMS: Secretaria Municipal de Saúde; SUS: Sistema Único de Saúde; UBS: unidades básicas de saúde.

conteúdo (12\%; 4/33). Algumas produções valeram-se de mais de uma metodologia, conforme disposto na Tabela 2 .

Dez artigos (30\%; 10/33) publicaram análises comparativas, sendo a mais frequente entre Administração Direta e Organização Social, empreendida em cinco artigos (15\%; 5/33).

Em 16 artigos (49\%; 16/33) foram utilizadas fontes secundárias, entre elas bibliografia, documentos e bases públicas de dados demográficos e administrativos. Já três artigos (9\%; 3/33) usaram apenas fontes primárias, por meio de abordagens qualitativas, entrevistas ou grupos focais. Em outros 14 artigos $(42 \% ; 14 / 33)$ constam tanto fontes primárias quanto secundárias.

\section{Discussão}

Parte da literatura analisada busca a explicação das causas da emergência e da difusão dos modelos alternativos de gestão de serviços públicos de saúde. Para isso, autores percorrem múltiplas abordagens, dos postulados do neoliberalismo e das reformas do aparelho do Estado à apresentação dos instrumentos da administração privada como condutores de pior ou melhor desempenho dos serviços de saúde. Os modelos de gestão são apresentados ora como a modernização ora como a destituição do papel e das atribuições do Estado na saúde.

A primeira explicação, exógena e de natureza macropolítica, localiza-se no cenário de privatizações 7 e transferências de responsabilidades do Estado para o setor privado, com gradativo recrudescimento das políticas públicas e desmonte do aparato estatal 8. Mas também está inserido no cenário da reforma gerencial, que previa transferir a oferta de serviços públicos para OS e provedores públicos não estatais, que passariam a receber recursos do Estado, com atuação regida por contrato de gestão. Outros objetivos eram a existência de agências reguladoras, a gestão baseada em resultados e a premiação por mérito, sob o argumento de tornar mais eficiente a administração pública, racionalizar os gastos e oferecer aos cidadãos serviços de melhor qualidade, atribuindo maior importância aos resultados financeiros e à produção dos serviços 9,10 .

A segunda motivação, endógena à gestão, foi uma resposta às dificuldades da Administração Direta em gerir adequadamente serviços de saúde, devido à sua baixa capacidade operacional, falta de autonomia orçamentária, financeira e administrativa, baixos controles de qualidade, influência política externa, burocracia excessiva para contratar pessoal, efetuar adequações de estrutura e comprar insumos, medicamentos e equipamentos. Somam-se as normas e procedimentos típicos da Administração Direta e a Lei de Responsabilidade Fiscal, que limitavam a expansão e qualificação do quadro de recursos humanos 11,12,13.

A literatura revisada também tratou de diferenças entre as modalidades de gestão, com base nos dados empíricos produzidos nos estudos e considerando as configurações jurídicas e os marcos legais constituintes. 


\section{Tabela 2}

Publicações selecionadas segundo procedimentos metodológicos utilizados.

\begin{tabular}{|c|c|c|c|c|}
\hline $\begin{array}{l}\text { Desenhos de } \\
\text { pesquisa }\end{array}$ & Estudo & Fontes de dados & Análise & Participantes \\
\hline \multirow[t]{11}{*}{ Estudo descritivo } & $\begin{array}{c}\text { Contreiras \& Matta } \\
\text { (2015) } 7\end{array}$ & $\begin{array}{c}\text { Secundárias (revisão bibliográfica e } \\
\text { análise documental) }\end{array}$ & $\begin{array}{l}\text { Descritiva e análise } \\
\text { de regulação }\end{array}$ & $\begin{array}{l}20 \text { entidades privadas } \\
\text { contratadas }\end{array}$ \\
\hline & Mendes et al. (2014) 45 & $\begin{array}{l}\text { Primárias (coleta de dados } \\
\text { demográficos, fluxos de } \\
\text { encaminhamento, tempo de espera, } \\
\text { custos, evolução médica) }\end{array}$ & Descritiva & 57 pacientes \\
\hline & Santos et al. (2014) 29 & Secundárias (Pesquisa AMS/IBGE) & Descritiva & $\begin{array}{c}97.449 \text { estabelecimentos } \\
\text { de saúde da Pesquisa } \\
\text { AMS/IBGE }\end{array}$ \\
\hline & Andreazzi (2013) 47 & $\begin{array}{c}\text { Secundárias (revisão bibliográfica e } \\
\text { análise documental) }\end{array}$ & Descritiva & NA \\
\hline & Camargo et al. (2013) 34 & Secundárias (análise documental) & $\begin{array}{l}\text { Construção de matriz } \\
\text { de indicadores }\end{array}$ & $\mathrm{NI}$ \\
\hline & Kaliks et al. (2013) 46 & $\begin{array}{l}\text { Primárias (coleta de dados } \\
\text { clínicos, intervalos entre exames } \\
\text { e procedimentos); Secundárias } \\
\text { (análise documental, dados } \\
\text { administrativos) }\end{array}$ & Descritiva & 51 pacientes \\
\hline & Sodré et al. (2013) 32 & $\begin{array}{c}\text { Secundárias (revisão bibliográfica e } \\
\text { análise documental) }\end{array}$ & Descritiva & NA \\
\hline & Puccini (2011) 31 & Secundárias (análise documental) & $\begin{array}{l}\text { Comparativa e } \\
\text { análise da regulação }\end{array}$ & NA \\
\hline & Barbosa (2012) 26 & Secundárias (análise documental) & Comparativa & 2 hospitais \\
\hline & Barbosa \& Elias (2010) 18 & $\begin{array}{l}\text { Primárias (entrevistas); Secundárias } \\
\text { (análise documental) }\end{array}$ & Comparativa & $\begin{array}{l}\text { Dirigentes e profissionais } \\
\text { dos } 2 \text { hospitais }\end{array}$ \\
\hline & Maciel et al. (2005) 48 & $\begin{array}{l}\text { Secundárias (dados administrativos } \\
\text { dos relatórios anuais do HCFMRP) }\end{array}$ & Comparativa & $\begin{array}{c}1 \text { hospital público e } \\
1 \text { fundação de apoio } \\
\text { privada }\end{array}$ \\
\hline \multirow[t]{5}{*}{ Pesquisa qualitativa } & Pacheco et al. (2016) 40 & $\begin{array}{c}\text { Primárias (entrevistas e grupo } \\
\text { focal); Secundárias (análise } \\
\text { documental) }\end{array}$ & $\begin{array}{c}\text { Análise de } \\
\text { condensação de } \\
\text { significados Kvale }\end{array}$ & $\begin{array}{c}18 \text { entrevistados: } \\
\text { gestores estaduais e } \\
\text { secretários municipais de } \\
\text { saúde }\end{array}$ \\
\hline & Barbosa \& Malik (2015) 30 & $\begin{array}{c}\text { Secundárias (revisão bibliográfica e } \\
\text { análise documental) }\end{array}$ & Análise crítica & 24 projetos de PPP \\
\hline & Romano et al. (2015) 41 & $\begin{array}{l}\text { Primárias (entrevistas); Secundárias } \\
\text { (análise documental) }\end{array}$ & Análise de conteúdo & $\begin{array}{c}6 \text { entrevistados: técnicos } \\
\text { da SES }\end{array}$ \\
\hline & Lorenzetti et al. (2014) 23 & $\begin{array}{c}\text { Primárias (entrevistas com } \\
\text { informantes-chave); Secundárias } \\
\text { (análise documental) }\end{array}$ & Comparativa & $\begin{array}{l}\text { Entrevistados: diretores } \\
\text { de hospitais de hospitais } \\
\text { privados, autoridades } \\
\text { executivas nas três } \\
\text { esferas públicas }\end{array}$ \\
\hline & Sano \& Abrucio (2008) 19 & $\begin{array}{c}\text { Primárias (entrevistas abertas); } \\
\text { Secundárias (revisão bibliográfica e } \\
\text { análise documental) }\end{array}$ & Descritiva & $\begin{array}{c}32 \text { entrevistados: } \\
\text { gestores, Legislativo, } \\
\text { Conselho Estadual de } \\
\text { Saúde, acadêmicos e } \\
\text { especialistas em gestão } \\
\text { hospitalar }\end{array}$ \\
\hline
\end{tabular}

(continua) 
Tabela 2 (continuação)

\begin{tabular}{|c|c|c|c|c|}
\hline $\begin{array}{l}\text { Desenhos de } \\
\text { pesquisa }\end{array}$ & Estudo & Fontes de dados & Análise & Participantes \\
\hline \multirow[t]{3}{*}{ Estudo de caso } & $\begin{array}{l}\text { Costa e Silva et al. } \\
\text { (2014) } 49\end{array}$ & $\begin{array}{l}\text { Primárias (entrevistas); Secundárias } \\
\text { (análise documental) }\end{array}$ & Comparativa & $\mathrm{NI}$ \\
\hline & $\begin{array}{l}\text { La Forgia \& Harding } \\
\qquad \text { (2009) } 36\end{array}$ & $\begin{array}{c}\text { Secundárias (dados administrativos } \\
\text { do DATASUS e CNES) }\end{array}$ & Quantitativa & $\begin{array}{l}12 \text { hospitais } \\
\text { administrados por } \\
\text { OSS e } 12 \text { hospitais } \\
\text { administrados por } \\
\text { Administração Direta }\end{array}$ \\
\hline & Quinhões (2009) 25 & $\begin{array}{l}\text { Primárias (entrevistas } \\
\text { semiestruturadas); Secundárias } \\
\text { (análise documental, dados } \\
\text { administrativos do SIH e SIA do } \\
\text { Ministério da Saúde, CNES) }\end{array}$ & Comparativa & $\begin{array}{c}3 \text { hospitais gerenciados } \\
\text { por OS, } 3 \text { hospitais } \\
\text { gerenciados por } \\
\text { Administração Direta, } \\
\text { entrevistados: diretores } \\
\text { de } 3 \text { hospitais, diretor da } \\
\text { CGCSS }\end{array}$ \\
\hline \multirow[t]{3}{*}{ Ensaio } & Anker \& Pereira (2013) 20 & $\begin{array}{l}\text { Secundárias (revisão bibliográfica e } \\
\text { análise documental) }\end{array}$ & Descritiva & 3 projetos de PPP \\
\hline & Nogueira (2011) 11 & $\begin{array}{l}\text { Secundárias (revisão bibliográfica, } \\
\text { análise documental, dados do } \\
\text { CNES) }\end{array}$ & Descritiva & NA \\
\hline & $\begin{array}{l}\text { Ibañez \& Vecina Neto } \\
\text { (2007) } 12\end{array}$ & $\begin{array}{c}\text { Secundárias (revisão bibliográfica e } \\
\text { análise documental) }\end{array}$ & Comparativa & NA \\
\hline \multirow[t]{2}{*}{ Revisão de literatura } & Ditterich et al. (2012) 24 & Secundárias (análise documental) & $\mathrm{NI}$ & $\mathrm{NI}$ \\
\hline & $\begin{array}{l}\text { Barata \& Mendes } \\
\text { (2007) } 43\end{array}$ & $\begin{array}{c}\text { Secundárias (revisão bibliográfica e } \\
\text { análise documental) }\end{array}$ & $\mathrm{NI}$ & $\mathrm{NI}$ \\
\hline Estudo exploratório & Lima \& Rivera (2012) 28 & $\begin{array}{l}\text { Primárias (entrevistas); Secundárias } \\
\text { (análise documental, dados } \\
\text { administrativos) }\end{array}$ & Análise de conteúdo & $\begin{array}{c}\text { Entrevistados: } 6 \\
\text { dirigentes e } 5 \text { gestores }\end{array}$ \\
\hline \multirow[t]{4}{*}{ Estudo de caso } & Cabral et al. (2016) 33 & $\begin{array}{c}\text { Primárias (entrevistas } \\
\text { semiestruturadas); Secundárias } \\
\text { (análise documental) }\end{array}$ & Comparativa & $\begin{array}{c}\text { Entrevistados: } 1 \\
\text { Secretário Estadual da } \\
\text { Fazenda, } 2 \text { assessoras } \\
\text { técnicas do setor da } \\
\text { saúde, } 1 \text { promotora } \\
\text { de Justiça do estado, } 1 \\
\text { presidente do consórcio } \\
\text { hospitalar e } 2 \text { gerentes } \\
\text { de bancos financiadores } \\
\text { dos projetos }\end{array}$ \\
\hline & $\begin{array}{l}\text { Costa e Silva et al. } \\
\qquad(2016) 27\end{array}$ & $\begin{array}{l}\text { Primárias (entrevistas); Secundárias } \\
\text { (análise documental) }\end{array}$ & $\begin{array}{c}\text { Análise de } \\
\text { implantação } \\
\text { do sistema de } \\
\text { governança }\end{array}$ & $\begin{array}{c}23 \text { entrevistados: } \\
\text { gerentes, coordenadores } \\
\text { e usuários }\end{array}$ \\
\hline & $\begin{array}{l}\text { Rodrigues \& Spagnuolo } \\
\qquad(2014) 42\end{array}$ & Primárias (entrevistas) & Análise de conteúdo & $\begin{array}{l}\text { Entrevistados: } 7 \\
\text { membros do Conselho } \\
\text { Gestor da OSS }\end{array}$ \\
\hline & André et al. (2013) 22 & $\begin{array}{c}\text { Primárias (entrevistas pelo método } \\
\text { Delphi) }\end{array}$ & $\begin{array}{l}\text { Quantitativa e } \\
\text { qualitativa }\end{array}$ & $\begin{array}{c}\text { Entrevistados: } 10 \\
\text { gestores de unidades de } \\
\text { saúde e } 10 \text { especialistas } \\
\text { da área de saúde }\end{array}$ \\
\hline
\end{tabular}

(continua) 
Tabela 2 (continuação)

\begin{tabular}{|c|c|c|c|c|}
\hline $\begin{array}{l}\text { Desenhos de } \\
\text { pesquisa }\end{array}$ & Estudo & Fontes de dados & Análise & Participantes \\
\hline \multirow[t]{3}{*}{$\begin{array}{l}\text { Pesquisa qualitativa/ } \\
\text { Estudo de caso }\end{array}$} & $\begin{array}{l}\text { La Forgia \& Couttolenc } \\
\qquad(2008) 50\end{array}$ & $\begin{array}{l}\text { Secundárias (dados administrativos } \\
\text { da SES, DATASUS e CNES) }\end{array}$ & Comparativa & $\begin{array}{c}12 \text { hospitais } \\
\text { administrados por } \\
\text { Oss e } 10 \text { hospitais } \\
\text { administrados por } \\
\text { Administração Direta }\end{array}$ \\
\hline & Matzuda et al. (2008) 21 & $\begin{array}{l}\text { Primárias (entrevista em grupo } \\
\text { focal); Secundárias (dados } \\
\text { administrativos da SES, DATASUS } \\
\text { e CNES) }\end{array}$ & Descritiva & $\begin{array}{l}20 \text { hospitais públicos, } \\
\text { OSS, privados, públicos } \\
\text { com fundação. } \\
\text { Entrevistados: médicos e } \\
\text { enfermeiras }\end{array}$ \\
\hline & $\begin{array}{l}\text { Carneiro Junior \& Elias } \\
\qquad(2006) 35\end{array}$ & $\begin{array}{c}\text { Primárias (entrevistas com } \\
\text { informantes-chave); Secundárias } \\
\text { (análise documental) }\end{array}$ & Análise de conteúdo & $\begin{array}{c}2 \text { OSS gerenciadoras de } \\
\text { hospitais (um na zona } \\
\text { leste do município e } \\
\text { outro na Grande São } \\
\text { Paulo); } 14 \text { entrevistados: } \\
\text { direção, gerência, } \\
\text { profissionais de saúde, } \\
\text { usuários, conselheiros } \\
\text { de saúde, deputados } \\
\text { estaduais, secretários de } \\
\text { saúde e técnicos da SES }\end{array}$ \\
\hline $\begin{array}{l}\text { Estudo de caso/ } \\
\text { Estudo descritivo }\end{array}$ & Melo \& Almeida (2014) 44 & $\begin{array}{l}\text { Primárias (entrevista } \\
\text { semiestruturada); Secundárias } \\
\text { (revisão bibliográfica) }\end{array}$ & Descritiva & 1 entrevistado: gestor \\
\hline
\end{tabular}

AMS/IBGE: Pesquisa de Assistência Médico-Sanitária do Instituto Brasileiro de Geografia e Estatística; CGCSS: Coordenadoria de Gestão de Contratos de Serviços de Saúde da Secretaria Estadual de Saúde de São Paulo; CNES: Cadastro Nacional de Estabelecimentos de Saúde; DATASUS: Departamento de Informática do SUS; HCFMRP: Hospital das Clínicas da Faculdade de Medicina de Ribeirão Preto; NA: não aplicável; NI: não informado; OSS: Organização Social de Saúde; PPP: Parcerias Público-Privadas; SES: Secretaria Estadual de Saúde; SIA: Sistema de Informação Ambulatorial; SIH: Sistema de Informação Sistema de Informações Hospitalares.

Destacou-se que a Administração Direta obedece às normas que disciplinam o serviço público, a exemplo do art. 37 da Constituição Federal e da Lei Federal no 8.666, de 21 de junho de 1993 14. Já as OS, entidades estruturais da reforma gerencial do Estado de 199510 foram regulamentadas pela Lei Federal no 9.637, de 15 de maio de 1998 15, por legislações estaduais e municipais, e legitimadas pelo Supremo Tribunal Federal em 2015 16. A instituição das PPPs, que respeitam as normas do direito privado, ocorreu por meio da Lei Federal no 11.079, de 30 de dezembro de 2004 6. Sem legislação específica, há propostas em trâmite 17 no Legislativo Federal para regulamentar as Fundações Estatais como organismos da Administração Pública Indireta, e que atribuem a elas maior flexibilidade e autonomia do que as atuais autarquias e fundações públicas de direito público. As fundações estatais foram propostas pelo Projeto de Lei Complementar no 92/2007, que regulamenta o inciso XIX do art. 37 da Constituição Federal 11,12.

O processo de compras públicas é um dos elementos principais do normativo da Administração Pública, que estipula uma série de obrigações e ritos para se firmar um contrato ou realizar uma compra. Para a Administração Direta, a Lei no 8.666/1993 é aplicada em sua integridade, gerando menor flexibilidade 18. No caso da Fundação Estatal, organismo da Administração Indireta, supõe-se maior celeridade em função da edição de regulamento próprio para as licitações, ainda que regidas pela Lei no 8.666/1993 11. Em relação às OSS, percebe-se certa flexibilidade por não estarem submetidas às normas do direito público, com suas obrigações sendo estabelecidas com base em contratos de gestão 12,19. Por fim, as compras realizadas pelas PPPs não estão sujeitas às restrições impostas às demais modalidades 20,21 . 
Os formatos de contratação e remuneração de recursos humanos, e as políticas de meritocracia e de resultados são aspectos destacados por estudos da presente revisão que abordam modelos de gestão. Na Administração Direta, a contratação de pessoal deve seguir o disposto no art. 37 da Constituição Federal de 1988, que impõe a necessidade de se realizar concurso público com regime de estabilidade, com exceção dos cargos de livre nomeação e exoneração, o que por vezes gera dificuldades para a atualização dos funcionários 22,23. Essa obrigatoriedade, porém, é desfeita nos três demais modelos. Com algumas diferenças, todos podem realizar a contratação de pessoal via regime CLT 18,19,24,25,26. Além da flexibilização, destaca-se o resultado positivo na gestão de recursos humanos da OSS frente a Administração Direta nos casos em que se identificou mudança na cultura organizacional, com maior participação dos funcionários e responsabilização 27.

Em relação ao financiamento dos serviços, as Fundações são remuneradas em função da produção, com pagamentos geralmente atrelados a metas pré-acordadas 12,28. A remuneração por desempenho também é o instrumento para financiar as OSS, por meio de contrato de gestão 19,29. As PPPs também são financiadas por critérios e regras definidos durante a licitação 20,30.

Independentemente da modalidade de gestão há previsão de mecanismos formais de controle interno e externo, como supervisão pelo Ministério da Saúde, controle e avaliação pelo Tribunal de Contas da União, dos estados e municípios e Ministério Público, subordinação ao controle social desempenhado pelos Conselhos de Saúde e obrigações de publicização de decisões, gastos e contratos 12 . As OSS e PPPs são constrangidas por contratos de gestão e mensuração de desempenho. Desde que estejam acordadas metas, instrumentos de acompanhamento, critérios objetivos para avaliar a produção e limites orçamentários, em tese é possível avaliar e cobrar as OS por melhores desempenhos 19. Já as PPPs são apresentadas como portadoras de um modelo diferenciado de controle, por meio do "estabelecimento de indicadores de desempenho output-bases, ou seja, baseados em metas e resultados (...) apurados a posteriori” 20 (p. 163). A remuneração pelos serviços prestados ocorreria apenas após auferido o alcance do que foi estipulado em contrato.

Apesar dos mecanismos formais de controle e avaliação de desempenho, a literatura analisada aponta para falhas no processo de delegação dos serviços públicos de saúde à gestão não realizada pela Administração Direta. No caso das OSS, aponta-se que a retórica do benefício do gerencialismo possa deixar de considerar os interesses privados que passam a atuar na gestão da saúde 31. Sobre as PPPs, a literatura indicou, com base em discussão descritiva e conceitual 32 e de interação com atores relevantes na implementação deste modelo 33, a capacidade do setor privado de influenciar e viabilizar projetos de seu interesse visando à obtenção de lucros.

Mesmo ao tratar de casos concretos, na produção analisada predominou a perspectiva conceitual do modelo, o arcabouço normativo, os regimes jurídicos, as práticas gerenciais e as relações contratuais. Mas houve também preocupação, em menor número de estudos, em apontar críticas à falta de transparência na prestação de contas pelas OSS 34, a expor interesses privados envolvidos na gestão dos serviços 31 e a questionar a suposta ampliação do acesso da população aos serviços, proporcionada por esses modelos 35 .

Não há consenso entre os poucos estudos comparativos que buscaram mencionar a superioridade de um modelo sobre o outro. Hospitais públicos geridos por OSS, quando comparados a hospitais geridos pela Administração Direta, têm maior eficiência econômica e melhor gestão de recursos humanos 12,19, além de menor gasto por leito hospitalar 36. Mas os serviços de São Paulo administrados por OSS apresentaram performance similar a dos serviços de Curitiba (Paraná) sob gestão da Administração Direta 21. Quanto ao desempenho de recursos humanos, as OSS, em função da flexibilidade na contratação e remuneração 18, registraram maior qualificação da força de trabalho 27. Outro estudo indicou não haver, entre OSS e Administração Direta, diferença na gestão de recursos humanos 21. As PPPs e Fundações Estatais, talvez pelo fato de serem pouco difundidas, nem sequer foram objeto de comparação com outros modelos.

Além de poucas, as comparações de modelos são frágeis na literatura analisada. Apenas um estudo realizou avaliações econômicas comparando duas ou mais alternativas, avaliando simultaneamente os custos e resultados 36. Não foram implementadas, no universo de artigos considerados, avaliações de qualidade ou comparativas da gestão dos serviços de saúde geridos por diferentes modelos que considerassem atributos como os de acessibilidade, equidade, satisfação e eficiência. 
Também não foram estudados possíveis impasses da convivência de distintos modelos na mesma rede, as repercussões da coexistência de padrões heterogêneos de gestão de pessoas, de serviços e remuneração, o que pode impactar a organização e o funcionamento do sistema de saúde como um todo, ou mesmo dificultar a configuração de rede integrada e serviços articulados em níveis de complexidade crescente, com a finalidade de garantir a integralidade da assistência à saúde em cada região delimitada.

E, ainda, não foram identificados nos estudos a realização de revisão sistemática ou narrativa da literatura, nem a utilização de surveys com gestores, usuários e profissionais de saúde, para citar dois exemplos de procedimentos metodológicos que poderiam ter sido acionados para analisar mais a fundo determinado modelo ou para comparar uma modalidade com outra.

A revisão demonstrou extrema concentração de estudos no Estado de São Paulo, com 18 publicações (55\%; 18/33). Segundo a Pesquisa de Assistência Médico-Sanitária do Instituto Brasileiro de Geografia e Estatística (IBGE. http://www.ibge.gov.br/home/estatistica/populacao/condicaodevida/ ams/2009/, acessado em 18/Jun/2017), em 2009, dentre os 94.070 estabelecimentos de saúde do Brasil, públicos ou privados, com ou sem fins lucrativos, em regime ambulatorial ou de internação, cerca de 15\% deles (14.215) estavam localizados no Estado de São Paulo. Considerando-se a disseminação, por todo o país, de modalidades de gestão de serviços de saúde alternativas à Administração Direta, a concentração dos estudos em São Paulo não acompanha a distribuição dos estabelecimentos no território nacional, deixando de explorar a possível diversidade de expressão e de desfechos dos modelos.

Com predominância de estudos qualitativos, revelou-se frágil a descrição da metodologia da maior parte dos estudos selecionados, e algumas análises descritivas e de conteúdo implementadas não produziram conclusões ou mesmo inferências interpretativas. É limitado o conjunto de referenciais teórico-metodológicos usados nos artigos considerados, o que revela situação similar à já apontada por Deslandes \& Iriart 37, que, ao analisarem o repertório de metodologias utilizadas na produção científica das ciências sociais e humanas em saúde, concluíram que há "monotonia e dominância de poucos tipos de modalidades de análise e de técnicas de produção de dados, reduzindo sobremaneira o amplo arsenal de escolhas e os novos e inovadores investimentos no percurso investigativo" 37 (p. 2385).

À semelhança de revisões da literatura estrangeira 38,39, a presente revisão demonstrou que também são escassas na produção científica nacional evidências do real impacto, na gestão de serviços de saúde, de modelos alternativos ou de arranjos entre público e privado. Não está comprovado que um modelo é intrinsicamente mais eficiente ou superior a outro, embora estudos de caso isolados atribuam, à modalidade de gestão, resultados em determinados serviços ou em contextos específicos.

As análises que comparam aspectos ou atributos de eficiência ou qualidade de modelos (públicos, privados com e sem fins lucrativos, ou mistos) de gestão em saúde no mundo concluíram que as diferenças são pouco significativas ou inconclusivas 18,21,36. A eficiência da prestação de serviços, seja qual for a natureza da gestão, dependerá das características do sistema de saúde e de fatores como financiamento, regulação, controle social externo, grau de monitoramento público sobre a execução dos serviços conveniados ou contratados, competição, desenvolvimento institucional, políticas de contratação e de remuneração de pessoal.

\section{Conclusão}

Conclui-se que a literatura nacional sobre modelos de gestão de serviços no SUS produziu até o momento um número restrito de artigos publicados, é concentrada geograficamente, principalmente no Estado de São Paulo, traz poucos estudos de modalidades introduzidas mais recentemente ou menos difundidas e assimiladas, é marcada pela baixa diversidade de teorias e por fragilidades de aportes metodológicos, dá ênfase a marcos legais e configurações jurídicas, prioriza os processos de contratualização, conveniamento, compras, recursos humanos, financiamento e controle.

A revisão aponta para a necessidade de produção científica que aprofunde o tema à altura de sua complexidade e abrangência, considerando que os modelos de gestão alternativos à Administração Direta são hoje hegemônicos na maior parte das redes públicas municipais e estaduais de saúde.

Dentre as abordagens que demandariam mais estudos estão os formatos societários, os trajetos de expansão da atuação e a acumulação de capital das organizações, entidades e empresas que atuam na 
gestão de serviços do SUS, bem como as redes de influência e os mecanismos por elas acionados para a preservação de seus interesses junto aos tomadores de decisão governamentais.

O presente trabalho também indica a importância de adotar outras abordagens metodológicas na literatura sobre o tema, de forma que possa alcançar pesquisas avaliativas capazes de comparar diferentes modelos e de lançar mão de procedimentos, métodos e instrumentos de pesquisa pelos quais a modalidade de gestão possa ser julgada pelo seu desempenho e seus efeitos na qualidade da assistência prestada, na saúde da população e na organização do sistema de saúde.

\section{Colaboradores}

A. F. Ravioli participou da concepção do trabalho, busca bibliográfica, elaboração e redação do artigo. P. C. De Soárez participou do desenho da pesquisa, análise de dados, redação e revisão final do artigo. M. C. Scheffer orientou a pesquisa, revisou criticamente o trabalho e aprovou a versão final do artigo.

\section{Agradecimentos}

Agradecemos à Coordenação do Departamento de Medicina Preventiva da Faculdade de Medicina da Universidade de São Paulo, que possibilitou a realização desta pesquisa no âmbito do Mestrado em Política, Planejamento e Gestão em Saúde. Ao $\mathrm{CNPq}$ pelo financiamento (bolsa de pesquisa do Projeto CNPq no 405077/2013-0).

\section{Referências}

1. Brasil. Lei Federal no 8.080, de 19 de setembro de 1990. Dispõe sobre as condições para a promoção, proteção e recuperação da saúde, a organização e o funcionamento dos serviços correspondentes e dá outras providências. Diário Oficial da União 1990; 20 set.

2. Scheffer MC, Aith FMA. O sistema de saúde brasileiro. Clín Méd 2016; 1:355-65.

3. Paim J, Travassos C, Almeida C, Bahia L, Macinko J. The Brazilian health system: history, advances, and challenges. Lancet 2011; 377:1778-97.

4. Santos JS, Teixeira CF. Política de saúde no Brasil: produção científica 1988-2014. Saúde Debate 2016; 40:219-30.

5. Green BN, Johnson CD, Adams A. Writing narrative literature reviews for peer-reviewed journals: secrets of the trade. J Chiropr Med 2006; 5:101-17.

6. Brasil. Lei no 11.079 , de 30 de dezembro de 2004. Institui normas gerais para licitação e contratação de parceria público-privada no âmbito da administração pública. Diário Oficial da União 2004; 31 dez.

7. Contreiras H, Matta GC. Privatização da gestão do sistema municipal de saúde por meio de Organizações Sociais na cidade de São Paulo, Brasil: caracterização e análise da regulação. Cad Saúde Pública 2015; 31:285-97.

8. Bravo MIS. Política social de saúde no Brasil. In: Mota AES, Bravo MIS, Teixeira M, Uchôa R, Marsiglia RMG, Gomes L, organizadores. Serviço social e saúde: formação e trabalho profissional. São Paulo: Cortez Editora; 2006. p. 1-24.

9. Bresser-Pereira LC. A reforma do estado dos anos 90: lógica e mecanismos de controle. Brasília: Ministério da Administração Federal e Reforma do Estado; 1997.

10. Bresser-Pereira LC. Reforma gerencial e legitimação do estado social. Rev Adm Pública 2017; 51:147-56.

11. Nogueira RP. O desenvolvimento federativo do SUS e as novas modalidades institucionais de gerência das unidades assistenciais. In: Santos NR, Amarante ODC, organizadores. Gestão pública e relação público privado na saúde. Rio de Janeiro: Centro Brasileiro de Estudos de Saúde; 2011. p. 24-47. 
12. Ibañez N, Vecina Neto G. Modelos de gestão e o SUS. Ciênc Saúde Coletiva 2007; 12:1831-40.

13. Couttolenc B, La Forgia G, Matsuda Y. Raising the quality of public spending and resource. Management in the health sector. https://openknowledge.worldbank.org/han dle/10986/10292 (acessado em 25/Jun/2017).

14. Brasil. Lei no 8.666, de 21 de junho de 1993. Regulamenta o art. 37, inciso XXI, da Constituição Federal, institui normas para licitações e contratos da Administração Pública e dá outras providências. Diário Oficial da União 1993; 22 jun.

15. Brasil. Lei no 9.637, de 15 de maio de 1998 . Dispõe sobre a qualificação de entidades como organizações sociais, a criação do Programa Nacional de Publicização, a extinção dos órgãos e entidades que menciona e a absorção de suas atividades por organizações sociais, e dá outras providências. Diário Oficial da União 1998; 18 mai.

16. Supremo Tribunal Federal. Ação Direta de Inconstitucionalidade no 1.923. http://www. stf.jus.br/portal/processo/verProcessoAnda mento.asp? numero $=1923 \&$ classe $=$ ADI \& origem $=\mathrm{AP} \&$ recurso $=0 \&$ tipoJulgamento $=\mathrm{M}$ (acessado em 25/Jun/2017).

17. Brasil. Projeto Fundação Estatal - principais aspectos. Brasília: Ministério do Planejamento, Orçamento e Gestão; 2007.

18. Barbosa NB, Elias PEM. As organizações sociais de saúde como forma de gestão público/privado. Ciênc Saúde Coletiva 2010; 15:2483-95.

19. Sano H, Abrucio FL. Promessas e resultados da nova gestão pública no Brasil: o caso das organizações sociais de saúde em São Paulo. Revista de Administração de Empresas 2008; 48:64-80.

20. Anker T, Pereira BR. O atual cenário das PPPs no setor de saúde pública no Brasil: potencialidades, desafios e as primeiras experiências em âmbito estadual. In: Oliveira G, Oliveira Filho LC, organizadores. Parcerias público-privadas: experiências, desafios e propostas. São Paulo: LTC; 2013. p. 159-80.

21. Matzuda Y, Rinne J, Shepherd G, Wenceslau J. Enhancing performance in Brazil's health sector: lessons from innovations in the State of São Paulo and the city of Curitiba. http://documents.worldbank.org/curated/ pt/664271468016810488/Brazil-Enhancingperformance-in-Brazils-health-sector-les sons-from-innovations-in-the-state-of-SaoPaulo-and-the-city-of-Curitiba (acessado em 25/Jun/2017).

22. André AM, Ciampone MHT, Santelle O. Tendências de gerenciamento de unidades de saúde e de pessoas. Rev Saúde Pública 2013; 47:158-63.

23. Lorenzetti J, Lanzoni GMM, Assuiti LFCA, Pires DEP, Ramos FRS. Gestão em saúde no Brasil: diálogo com gestores públicos e privados. Texto \& Contexto Enferm 2014; 23:417-25.

24. Ditterich RG, Moysés ST, Moysés SJ. O uso de contratos de gestão e incentivos profissionais no setor público de saúde. Cad Saúde Pública 2012; 28:615-25.
25. Quinhões TAT. O modelo de governança das Organizações Sociais de Saúde (OSS) e a qualidade do gasto público hospitalar corrente. In: XIV Prêmio Tesouro Nacional 2009. http:// www3.tesouro.fazenda.gov.br/Premio_TN/ XIVPremio/qualidade/1qualidadeXIVPTN/ Monografia_Tema4_Trajano_Augustus.pdf (acessado em 25/Jun/2017).

26. Barbosa NB. Regulação do trabalho no contexto das novas relações público versus privado na saúde. Ciênc Saúde Coletiva 2012; 15:2497506.

27. Costa e Silva V, Barbosa PR, Hortale VA. Parcerias na saúde: as Organizações Sociais como limites e possibilidades na gerência da Estratégia Saúde da Família. Ciênc Saúde Coletiva 2016; 21:1365-76.

28. Lima SML, Rivera FJU. A contratualização nos hospitais de ensino no Sistema Único de Saúde brasileiro. Ciênc Saúde Coletiva 2012; 17:2507-21.

29. Santos MAB, Madeira FC, Passos SRL, Bakr F, Oliveira KB, Andreazzi MAR. Autonomia financeira em estabelecimentos públicos e privados de saúde no Brasil. Cad Saúde Pública 2014; 30:201-6.

30. Barbosa AP, Malik AM. Desafios na organização de parcerias público-privadas em saúde no Brasil. Análise de projetos estruturados entre janeiro de 2010 e março de 2014. Rev Adm Pública 2015; 49:1143-65.

31. Puccini PT. As Organizações Sociais e o direito à saúde. http://idisa.org.br/img/File/ SUS\%20OS\%20Paulo\%20Puccini\%20Fim\%20 6_4_2011.pdf (acessado em 11/Mar/2018).

32. Sodré F, Littike D, Drago LMB, Perim MCM. Empresa Brasileira de Serviços Hospitalares: um novo modelo de gestão? Serv Soc Soc 2013; 114:365-80.

33. Cabral S, Fernandes ASA, Ribeiro DBC. Os papéis dos stakeholders na implementação das parcerias público-privadas no Estado da Bahia. Cadernos EBAPE.BR 2016; 14:325-39.

34. Camargo TA, Prado O, Bernardo R, Lara ACHS. Controle e transparência no modelo de organizações sociais de saúde do Estado de São Paulo. In: VI Congresso Consad de Gestão Pública 2013. http://www.escoladegestao. pr.gov.br/arquivos/File/2013/V_CONSAD/ VI_CONSAD/050.pdf (acessado em 25/Jun/ 2017).

35. Carneiro Junior N, Elias PEM. Controle público e equidade no acesso a hospitais sob gestão pública não estatal. Rev Saúde Pública 2006; 40:914-20.

36. La Forgia GM, Harding A. Public-private partnerships and public hospital performance in São Paulo, Brazil. Health Aff (Millwood) 2009; 28:1114-26.

37. Deslandes SF, Iriart JAB. Usos teórico-metodológicos das pesquisas na área de Ciências Sociais e Humanas em Saúde. Cad Saúde Pública 2012; 28:2380-6. 
38. Basu S, Andrews J, Kishore S, Panjabi R, Stuckler D. Comparative performance of private and public healthcare systems in low- and middle-income countries: a systematic review. PLoS Med 2012; 9:e1001244.

39. Rao S. Is the private sector more efficient? A cautionary tale. http://www.gsdrc.org/doc ument-library/is-the-private-sector-moreefficient-a-cautionary-tale/ (acessado em 25/ Jun/2017).

40. Pacheco HF, Gurgel Júnior GD, Santos FAS, Ferreira SCC, Leal EMM. Organizações sociais como modelo de gestão da assistência à saúde em Pernambuco, Brasil: percepção de gestores. Interface (Botucatu, Online) 2016; 20:585-95.

41. Romano CMC, Scatena JHG, Kehrig RT. Articulação público-privada na atenção ambulatorial de média e alta complexidade do SUS: atuação da Secretaria de Estado de Saúde de Mato Grosso. Physis (Rio J.) 2015; 25:1095115.

42. Rodrigues CT, Spagnuolo RS. Organizações Sociais de Saúde: potencialidades e limites na gestão pública. Rev Eletrônica Enferm 2014; 16:549-57.

43. Barata LRB, Mendes JDV. Organizações Sociais de Saúde: a experiência exitosa de gestão pública de saúde do Estado de São Paulo. São Paulo: Secretaria Estadual de Saúde; 2007.

44. Melo TBN, Almeida MR. Parceria público-privada: a gestão privada em hospitais universitários no Rio Grande do Norte. Revista de Saúde Pública de Santa Catarina 2014; 7:46-62.
45. Mendes CA, Martins AA, Teivelis MP, Kuzniec S, Wolosker N. Parceria público-privada em cirurgia vascular. Einstein (São Paulo) 2014; 12:342-6.

46. Kaliks RA, Pontes LB, Bognar CLFB, Santos KCC, Bromberg SE, Amaral PGT, et al. Pacientes com câncer de mama oriundas do Sistema Único de Saúde tratadas no setor privado: custos de um piloto de parceria público-privada em oncologia. Einstein (São Paulo) 2013; 11:216-23.

47. Andreazzi MFS. Empresa Brasileira de Serviços Hospitalares: inconsistências à luz da reforma do Estado. Rev Bras Educ Méd 2013; 37:275-84.

48. Maciel BC, Wichert MAAL, Peroni SP, Próspero UOS. Gestão em parceria entre uma fundação de apoio e um hospital público universitário: análise custo-efetividade. Revista de Administração 2005; 40:342-52.

49. Costa e Silva V, Escoval A, Hortale VA. Contratualização na Atenção Primária à Saúde: a experiência de Portugal e Brasil. Ciênc Saúde Coletiva 2014; 19:3593-604.

50. La Forgia GM, Couttolenc BF. Hospital performance in Brazil: the search for excellence. https://openknowledge.worldbank.org/han dle/10986/10284 (acessado em 25/Jun/2017). 


\section{Abstract}

The current study aimed to systematically analyze trends and priorities in the theoretical and conceptual approaches and empirical studies on specific health services management modalities in the Brazilian Unified National Health System. A narrative review of the literature identified, in 33 publications, the location and nature of services, management models, methodological procedures, and study outcomes. The research deals mainly with the models' conceptual and legal characteristics and management practices, in addition to addressing contracts, procurement, human resources, financing, and control mechanisms. In conclusion, the literature is limited and concentrated in the State of São Paulo, showing little theoretical diversity and methodological weaknesses, while it is nonconclusive as to the superiority of one management model over another. New evaluation studies are needed that are capable of comparing different models and assessing their performance and their effects on the quality of health services' provision, the population's health, and the health system's organization.

Health Management; Health Services Administration; Unified Health System

\section{Resumen}

El presente estudio tuvo como objetivo analizar sistemáticamente las tendencias y prioridades en los enfoques teóricos y conceptuales y estudios empíricos sobre modalidades específicas de gestión de los servicios de salud en el Sistema Único de Salud de Brasil. Una revisión narrativa de la literatura identificó, en 33 publicaciones, la ubicación y la naturaleza de los servicios, los modelos de gestión, los procedimientos metodológicos y los resultados del estudio. La investigación se ocupa principalmente de las características conceptuales y legales $y$ las prácticas de gestión de los modelos, además de abordar los contratos, las adquisiciones, los recursos humanos, el financiamiento y los mecanismos de control. En conclusión, la literatura es limitada $y$ concentrada en el Estado de São Paulo, mostrando poca diversidad teórica y debilidades metodológicas, mientras que no es concluyente en cuanto a la superioridad de un modelo de gestión sobre otro. Se necesitan nuevos estudios de evaluación que sean capaces de comparar diferentes modelos $y$ evaluar su desempeño y sus efectos sobre la calidad de la provisión de servicios de salud, la salud de la población y la organización del sistema de salud.

Gestión en Salud; Administración de los Servicios de Salud; Sistema Único de Salud
Recebido em 04/Jul/2017

Versão final reapresentada em 12/Jan/2018

Aprovado em 26/Jan/2018 